Compte rendu de Le tatouage samoan. Un rite polynésien dans l'histoire de Sébastien Galliot

\title{
Sophie Chave-Dartoen
}

\section{OpenEdition}

Journals

Édition électronique

URL : https://journals.openedition.org/jso/12775

DOI : 10.4000/jso. 12775

ISBN : $1760-7256$

ISSN : 1760-7256

Éditeur

Société des océanistes

Édition imprimée

Date de publication : 30 juin 2021

Pagination : 176-181

ISBN : 978-2-85430-140-3

ISSN : 0300-953x

Référence électronique

Sophie Chave-Dartoen, "compte rendu de Le tatouage samoan. Un rite polynésien dans l'histoire de Sébastien Galliot », Journal de la Société des Océanistes [En ligne], 152 | 2021, mis en ligne le 30 juin 2021, consulté le 03 janvier 2023. URL : http://journals.openedition.org/jso/12775 ; DOI : https://doi.org/ $10.4000 /$ jso. 12775

\section{(c)}

Creative Commons - Attribution - Pas d'Utilisation Commerciale - Pas de Modification 4.0 International - CC BY-NC-ND 4.0

https://creativecommons.org/licenses/by-nc-nd/4.0/ 
lose it power once removed from ritual contexts, and the importance of women's creative forms. Stanley concludes with the statement that these questions will be answered in the final chapter, by which he means the Conclusion.

Chapter 8 is called "Visual adventures." While most of the previous sections have focused on the influences on Asmat carving, here Stanley discusses the impact of Asmat art on global creative expression. Specific examples are noted in regards to documentary film and still photography that still fall within the category of documentation. In contrast are what Stanley calls "artistic surveyors" who break from the documentarians via a "self-reflexiveness in their actions that breaks the iron laws of primordialism (p. 179)." Among these are filmmakers Paul Carter and Roy Villevoye, drawer Pet Bognor, photographer Wim van Oijen, and painters Fons Bloemen and Ingo Wegerl. Stanley does not provide much context concerning the various studies of cultural appropriation. However, as his focus in specifically on the Asmat, this absence does not significantly weaken his arguments.

Stanley's Conclusion, titled "Dazzling the anthropologists" discusses why anthropologists, missionaries, and the world at large have been so taken with Asmat carving. Here he delves into what is closer to philosophy than the history centered previous chapters. Is it the aesthetics, the strength of design, the intriguing iconography that hold the viewer captive? However, at the end he returns to the issue of who is the evaluator and what is being evaluated. Who is influencing whom? Yet, the questions asked in Chapter 7 are never really answered and maybe cannot be. For perhaps the greatest problem in the book is not one of Stanley's making, but a result of the existing corpus of work on the Asmat. That is the absence of the thoughts and perceptions of the people themselves. This is not a problem of which Stanley is unaware. He notes this concern from scholars, missionaries, and others throughout the book. That is "What is it the Asmat think?"

Other problems in the book are not the result of philosophical problems or a lack of sources. One difficulty is the large number of typographic and grammatical errors in the text. For example, we have the unfortunate situation on page 90 where Gerbrands "bought" his colleague Philip Dark, rather than brought him to visit the Asmat. Another example is the difference in the titles presented for Chapter 2. The layout is inconsistent as well, with numerous places where the first word of the next sentence immediately follows a period, with no space between.

Despite its gaps, and even the typos, The Making of Asmat Art, is an important addition to the study of the Asmat and to the consideration of global forces at work in the acceptance and dissemination of indigenous art. In his exposition on the Asmat, Stanley has demonstrated the connectedness of the world today. By looking closely at the history of a once isolated people, he challenges the reader to think about art, culture, and history in a holistic way. The process can be messy and may not result in definitive answers, but the questions he raises are important and deserving of consideration.

Anne E. Guernsey Allen, Professor of Fine Arts, Indiana University, Southeast

Galliot Sébastien, 2019. Le tatouage samoan. Un rite polynésien dans l'histoire, Paris, CNRs éditions, 447 p., bibliogr, ill., annexes.

$S$ il est un lieu, dans le Pacifique, où la pratique du tatouage s'est perpétuée sans rupture de continuité, c'est bien l'archipel des Samoa occidentales, auquel Sébastien Galliot consacre la plus grande part de sa thèse de doctorat ${ }^{1}$. Il restitue son travail monographique dans un livre dont le sous-titre montre toute l'ambition: un rite polynésien dans l'histoire.

L'étude est donc historique, non qu'elle retrace l'histoire du tatouage à Samoa, mais parce qu'elle considère l'actualité des pratiques dans la complexité de leur développement, de leurs significations, de leurs réalisations. Ainsi, le livre commence-t-il assez classiquement par les traditions orales anciennes associées au tatouage, l'histoire des contacts et de l'installation des missions (les trois premiers chapitres), pour s'attacher à la spécificité des usages samoans. L'analyse commence par la discussion des théories qu'Alfred Gell défend dans Wrapping in images (chap. 4) pour s'étendre aux praticiens, aux pratiques, aux objets, aux images (chapitres 5 à 8), avant une "Discussion sur les savoirs rituels et leur transmission » (chap. 9) que vient clore une conclusion générale (439 pages en tout, illustrations et bibliographie comprises).

Précisons tout de suite que, dans sa pratique «traditionnelle ", le tatouage samoan est une institution rituelle, et au premier chef, une initiation sous la forme d'une "souffrance", une "douleur" partagée entre paires de co-initiés - qui sont en relation soa - et avec les autres accompagnants, chargés de la "prière " tāpuải qui assure la réussite d'une opération hautement dangereuse. De là, le tatouage tire toute son importance et sa signification pour les $\mathrm{Sa}$ moans. Bien qu'ils se soient considérablement simplifiés au fil du temps, on constate que

« les aspects techniques, esthétiques et économiques de l'activité de l'expert technico-rituel se situent dans un rapport d'interdépendance entre les communautés locales (urbaines et rurales), les diasporas polynésiennes, la "clientèle" occidentale et certains tatoueurs professionnels occidentaux » (p. 9)

1. Pour la thèse, et le présent livre qui en découle, le terrain fut réalisé dans les Samoa occidentales et dans la diaspora samoanne de Nouvelle-Zélande. 
L'auteur propose donc une contribution à l'«anthropologie du tatouage " dans une perspective centrée sur l'étude des " dispositifs matériels et relationnels, et de ses effets visuels et physiques sur les corps des participants » (p. 34). Il propose de délaisser les approches représentationnelles du rituel et sémiotiques de l'image pour privilégier "une approche technologique de l'efficacité rituelle» (pp. 34-39) qui s'étend aux relations qui lui donnent sens pour les acteurs concernés.

L'introduction générale explicite le sujet et la perspective retenus : c'est en tant qu'apprenti tatoueur que $S$. Galliot a conduit l'enquête. Il a accédé, avec leur plein accord, aux savoir-faire et au point de vue de praticiens engagés dans les responsabilités locales d'officiants rituels et très mobilisés, par ailleurs, sur la scène internationale où leur expertise et la virtuosité de leurs tatouages sont prisées. Ici, les tatouages rituels ( $p e ́ a$, malu, mälōfie ${ }^{2}$ ) considérés comme « traditionnels ", ont qualité de "bien de valeur ». Le rituel, en lui-même, opère des configurations spatiales et relationnelles où l'agencement des statuts sociaux (leur activation, leur expression, leur réorganisation) est central. Ces deux aspects se combinent, montre l'auteur, pour favoriser des "processus de subjectivation» passant par l'engagement du corps dans l'action, la manifestation de la bravoure du patient et le soutien des déités tutélaires convoquées pour l'occasion.

Car le tatouage est avant tout un rituel, et sa réalisation, marquée par des interdits. Étant "sacré" (sā, p. 249), il nécessite des connexions appropriées avec les déités (« divinités tutélaires » et/ou " esprits ancestraux ", aitu) dans différents registres : le premier, généalogique, lie le tatoueur et les dépositaires initiaux de la technique telle que, selon le mythe d'origine, elle fut rapportée de Fidji par deux déités féminines, les sœurs siamoises Taemā et Tilafaigā. Une fois séparées, l'une s'adonna à la guerre, l'autre au tatouage des hommes, le lien étant clairement énoncé entre la virilité, le tatouage et l'autre activité sanglante qu'est la guerre. De façon classique pour la Polynésie, chaque expert fait valoir une variante du mythe qui établit et garantit ce lien généalogique avec un esprit (aitu) qui " possède un réel pouvoir de mort sur ses patients " (p. 252). Le respect de ce lien garantit donc la réussite du tatouage et c'est avec les outils initialement légués par les déités que chaque expert opère, agissant « en lieu et place des esprits du tatouage " (p. 229). Notons que cette relation généalogique a aussi une dimension politique éminente, au moins parmi les tatoueurs puisque, rattachant chacun à un lignage localisé, elle établit sa légitimité, sa position statutaire relative et son autorité face à celles des autres.
Un autre registre de relation est interactionnel: la réussite du tatouage "dépend de l'entretien de bonnes relations avec les divinités " (p. 47) envers lesquelles les participants doivent remplir des obligations. Il s'agit aussi de se prémunir des " esprits rôdeurs" (aitu) qui, attirés par le sang, "volent les âmes des imprudents" (p. 243). Notons, qu'outre divers interdits, le rite est accompagné de prières. Les unes sont formulées silencieusement lors du tāpua'i $i$ (on aurait aimé savoir ici à qui, plus précisément, ces prières silencieuses sont adressées ${ }^{3}$ ); d'autres sont formulées à haute voix, telle la bénédiction que le prêtre ou le pasteur prononcent lors de la clôture du rite, juste avant la désacralisation des tatoués et la rétribution finale du tatoueur et de ses assistants. Les interactions verbales constantes entre les membres du tāpuải et la paire tatoueur/tatoué (seul le tatoueur répondant aux encouragements émanant du tāpuải) montrent que l'action est clairement distribuée et que son issue repose sur une conjonction d'efforts, non sur la seule réalisation technique.

Pris au sens d'institution sociale, le tatouage est aussi un rite d'initiation sanglant, collectif et public. Il était autrefois patronné par un chef de haut rang, figure ancestrale incarnée par le porteur vivant de son nom-titre. Ce dernier "commanditait " la cérémonie pour son fils et les jeunes gens du village invités à " partager la douleur" - c'est encore le cas pour certains dignitaires (un exemple détaillé dans le livre concerne un membre du gouvernement). Autrefois, le chef s'appuyait sur le vaste réseau de ses relations afin de rassembler les ressources matérielles considérables nécessaires à la réalisation du rite et à la rétribution du maitre tatoueur. Il confortait ainsi son autorité en favorisant l'accès des co-initiés au statut des jeunes adultes formant la «force du village " (auma$g a)$. La bravoure et la force physique de ces derniers s'exprimaient dans les activités sexuelles, cérémonielles et militaires, au service de la chefferie et pour sa défense, au temps des guerres inter-claniques. Ce faisant, le commanditaire s'assurait également de la participation des groupes de ces co-initiés aux futurs échanges cérémoniels réalisés sous sa direction.

Sébastien Galliot souligne donc que ce rite de passage

« relève aussi d'une technologie du pouvoir - au sens foucaldien - visant la construction de sujets politiques par l'intervention sur le corps » (p. 28)

Il a une efficacité sociale en ce qu'il reconduit des rapports hiérarchiques constitutifs de la société, permet l'acquisition, par les jeunes gens, «des dispositions psychologiques nécessaires au maintien de l'intégrité économique et politique " de la communauté villageoise (p. 145). Chose essentielle selon lui, cette

2. Les tatouages pe’a (en forme de pantis recouvrant le dos et les reins pour descendre aux genoux) sont réservés aux hommes; les malu, moins couvrants et étendus sur les seules cuisses, sont réservés aux femmes. Mälōfie est un terme appartenant au vocabulaire réservé aux personnes de haut statut. Il désigne le tatouage masculin et féminin, notamment lors du rite final de « désacralisation » par onction d'huile et de curcuma.

3. La pratique du tāpuải, à Samoa, est bien décrite dans la littérature francophone (Tcherkezoff, 2003 ; Clément, 2014). Un interlocuteur de S. Galliot traduit le terme par « vénérer, idolâtrer » (p. 247). 
" construction des sujets et leur intégration en une catégorie de personnes au service de la chefferie [... repose sur des actes techniques » (p. 37)

Lanalyse, menée au présent, défend l'idée que les logiques anciennes ont perduré, malgré l'effacement relatif de l'autorité coutumière face à la religion et à d'autres formes d'affiliation. Cependant, l'auteur montre bien que la pratique échappe à toutes formes d'assujettissement pour les Samoans urbains ou émigrés, et relève de choix personnels au regard des opportunités, du soutien de la parentèle, de l'affiliation religieuse, de traditions familiales ou du respect envers les anciens (notamment s'ils sont, ou non, tatoués). Dans le cadre villageois, le tatouage garde dans une certaine mesure des fonctions sociales directement liées à l'organisation de la chefferie et aux dynamiques qui la sous-tendent. Ici, toutefois, l'excellente description du système social laisse voir une immense complexité qu'une étude menée dans le seul registre politique tendrait à réduire. En effet, tous les hommes étant autrefois tatoués, le tatouage n'établissait pas - ou peu - de distinction entre eux ${ }^{4}$. Tous étaient ainsi reconnus dans leur valeur virile et intégrés à la communauté avant même que ne leur incombe un éventuel titre de matai ou d'alii. Certes, en commanditant le tatouage des garçons d'une classe d'âge, les chefs s'attachaient des "dépendants " qui agissaient pour eux, tout comme eux-mêmes étaient en relation de dépendance envers des supérieurs. Lobligation ainsi créée était donc générale et allait dans les deux sens: donner un tatouage pour recevoir un service, quel que soit le statut de la personne, chef suprême excepté. Si des stratégies personnelles ou collectives visant l'exercice du pouvoir existaient et existent sans doute encore à Samoa, Sébastien Galliot montre bien que - en théorie tout au moins elles ne sont pas soumises à l'exercice direct d'une quelconque contrainte au sein du groupe social, mais à deux principes essentiels, toujours très vivaces, que la seule force ne confere pas. Le premier est la logique complexe de la gradation des titres conférant un statut à leurs porteurs respectifs et à leur groupe. Seule l'ancienneté d'un titre et son importance dans l'histoire établit la dignité (mamalu), le champ d'action (pule) et le degré de respect (fáaaloalo) reconnus à son porteur. Le second principe est le service (tautua), valeur fondatrice qu' exprime un adage connu : « la voie vers l'autorité, c'est le service! » Il est d'ailleurs rappelé à cet égard, que

« [L]e fait est bien connu à Samoa : l'exercice de l'autorité ne s'applique que dans la mesure où les hommes qui ne portent pas de titre (tauleléa) sont disposés à s'y soumettre » (pp. 195-196)

Le " pouvoir" - dans son acception tant classique que foucaldienne - semble donc moins central dans cette affaire que le service réciproque entre les chefs et leurs dépendants 5 . Certes, le jeu de l'ancienneté généalogique et positionnelle confère aux premiers des responsabilités d'ordre supérieur (ils officiaient autrefois dans la médiation avec les déités tutélaires) et opère donc une dissymétrie entre les pôles de la relation. D'un point de vue local, les relations en jeu ne relèvent donc pas d'une sujétion proprement politique, mais d'une conception particulière des responsabilités distribuées et du "service " que se doivent les membres de la communauté. Réciproque, mais orienté, ce dernier s'impose à tous, fondant l'autorité des chefs (et des maîtres tatoueurs ${ }^{6}$ ) avec la dignité et le respect que cela suppose. Pour cette raison,

«toutes les réunions formelles sont considérées comme davantage mamalu (dignes), si le divertissement (fáafiafia) et le service (tautua) sont accomplis par des soga'imiti (des hommes tatoués) et des jeunes femmes tatouées. [...] Le [tatouage féminin] malu participe donc de dispositifs cérémoniels, d'une esthétique de la présentation publique, d'une fertilité signalée et magnifiée par le tatouage" (p. 151)

Il en va de même pour une virilité pleinement réalisée telle que signalée par le tatouage masculin ( $p e ́ a)$ lors des offices cérémoniels. Bref, le tatouage semble moins assujettir hommes et femmes à la chefferie qu'il ne leur assure les conditions d'une interaction appropriée avec les chefs et les entités de l'au-delà que ces derniers "instancient" (Chave-Dartoen, 2013).

Bien que cela ne soit très explicitement dit qu'en fin d'ouvrage (p. 374), le tatouage apparait surtout comme une technologie rituelle d'enveloppement destinée au plein exercice des rites et des cérémonies que préside un chef : danse, service du kava et guerre comprise. Il confere une forme de complétude sociale et cérémonielle qui manifeste le pouvoir génésique des jeunes gens. L'auteur écarte pourtant de façon surprenante la possible «correspondance entre une zone tatouée et l'amélioration ou la mise en lumière des facultés de la partie du corps concernée " (p. 382). La fonction protectrice des enveloppements à Samoa, bien montrée dans les travaux de $\mathrm{S}$. Tcherkezoff sur le recouvrement et la protection de la vie $(2003,2013)$ n'est pas mentionnée. On apprend pourtant que le port du pagne est obligatoire pour assister au conseil des chefs, aux messes et à toutes les circonstances où on est « en relation

4. Si le tatouage des enfants de chefs pouvait être plus soigné ou plus élaboré que celui de leurs compagnons, c’était pour honorer leur père, les charges dans la chefferie n'étant pas nécessairement héréditaires en ligne directe (p. 233).

5. Il faudrait ici distinguer les relations internes au groupe, conçues et glosées en termes de service réciproque, et les relations entre groupes où la violence et la force pouvaient autrefois s'exercer avec, en cas de conflit, la domination des vainqueurs sur les vaincus : exil, exploitation des hommes, appropriation des femmes.

6. Le parallélisme entre chefs et experts tatoueurs s'étend à l'usage, dans les deux cas, de langages spécifiques en adresse et en référence, à l'organisation de cérémonies du kava particulières, au partage des responsabilités qui incombent à leurs épouses et au statut que cela confere à ces dernières. La position et le rôle de médiateurs avec les déités (et peut-être le Dieu chrétien, mais S. Galliot n’en parle pas) sont centraux dans ces deux cas. 
avec des ancêtres » (p. 364). Il est également précisé que le terme malu, nom du tatouage féminin, signifie "être protégé ", notamment des mauvais esprits (p. 380). On se serait attendu ici à une discussion de la théorie de Gell (1995) sur la "fermeture " (closure) que le tatouage réalise autour du bas-ventre et des organes de la génération, complétant jupes et pagnes pour préserver (tapu) la force vitale des hommes face aux ancêtres et aux déités. La permanence du tatouage pour les officiants samoans pourrait aussi s'expliquer par la manifestation, lors des cérémonies, d'une asymétrie fondamentale et par la préservation rituelle de ses dangers : la vulnérabilité des vivants et de leur forme d'existence (la vie et sa fertilité) face aux anciens (chefferie, ancêtres et déités, Dieu) et au domaine de l'au-delà devant lesquels ils se trouvent exposés. Tel est le cas, pour le port d'une natte cérémonielle à Wallis, par exemple (Chave-Dartoen, 1997). S. Galliot indique qu’à Samoa, le tatouage transforme l'enveloppe corporelle du patient, modifie son statut et ses dispositions à l'égard de la société, suscite des sentiments durables. Peut-être s'agit-il aussi d'acquérir la garantie d'une interaction appropriée avec ancêtres et déités ?

Il est probable que Sébastien Galliot s'est détourné de ce type d'analyse classique pour mettre en valeur l'approche novatrice qu'il propose, centrée sur les processus de subjectivation des personnes tatouées et des formes d'efficacité reconnues au tatouage comme technique de modification de l'enveloppe corporelle. Laction technique (et rituelle) du tatouage vient en effet transformer «la peau humaine, l'enveloppe corporelle, elle-même déjà modelée par la société et investie par le sujet» (p. 32), modifiant l'image du corps " par l'intervention conjointe des gestes, des substances et des participants au rituel». Certains dispositifs matériels affectent, en effet, «l'expérience sensorielle du sujet » (p. 32) et, avec sa capacité à supporter bravement la douleur, sa relation au monde. Cette entrée, où se combine construction sociale de la personne et élaboration singulière des sujets, est très convaincante. Elle permet de comprendre que, de nos jours, les enjeux identitaires sont devenus cruciaux, qu'il s'agisse de s'inscrire dans une lignée familiale, d'affirmer une affiliation ethnique et culturelle en contexte de migration, ou encore de marquer une étape dans le parcours de vie afin d'acquérir confiance en soi et une forme d'accomplissement essentiellement personnel. Attaché au tatouage rituel, cet ouvrage participe donc à l'étude des processus de subjectivation proprement polynésiens, par ailleurs, mal connus?. Ils sont principalement "politiques", explique S. Galliot, terme, on l'aura compris, à entendre comme le jeu complexe des relations sociales et des responsabilités organisées par le système des titres et des obligations mutuelles qu'il suppose - et non comme des relations de pouvoir fondées sur la contrainte et l'assujettissement unilatéral. Ces processus sont donc bien différents du narcissisme des "pratiques de soi » ou d' "écriture de soi » (p. 11), telles qu'elles sont généralement pensées dans le cadre d'un monde globalisé où circulent les images et les significations qui leurs sont associées. C'est pourquoi l'auteur écarte une "iconologie des marques et une sémiotique corporelle» (p. 9) telles que proposées dans la plupart des études sur le tatouage ainsi que toute théorie du rituel expressiviste ou " communicationnelle ». Il défend une approche qui, partant de l'étude technologique de la pratique, aborde outre ses dimensions relationnelles - ses effets physiques (la douleur, les démangeaisons, l'épaississement de la peau etc.). Ces derniers «doivent être analysés et intégrés à l'étude de la co-construction des sujets et des objets " que sont donc, ici, les tatouages rituels (p. 29). L'usage fait, à la fois descriptif et analytique, de la chaine opératoire est pleinement convaincant. Il restitue bien la complexité des opérations techniques plus ou moins étroitement enchaînées (confection des outils, préparation des pigments, séquences du tatouage...), montrant leurs dimensions collectives et coordonnées où la technique et le social sont étroitement imbriqués. Une approche technique du rituel présente ainsi une réelle vertu heuristique. L'ensemble de l'ouvrage plaide toutefois pour l'intégration des conceptions locales de l'action et de sa réussite à ce type d'analyse, la distribution de l'action intégrant des entités ancestrales et divines dont la présence est seule garantie du résultat.

Cette perspective, développée en toute fin d'ouvrage, éclaire l'approche résolument "post-sémiotique $^{8}$ » que revendique l'auteur. Ce qu'il faut retenir du tatouage samoan, selon Sébastien Galliot, c'est l'accomplissement « du processus technique de création d'une image » (p. 31) qui soit appropriée, réalisée dans des conditions précises par un expert rituel avec des outils spécifiques (p. 33). Cette proposition forte - et très stimulante - qui rejette tout intérêt pour l'image en elle-même, est développée tardivement dans l'ouvrage et ne reçoit peut-être pas tout le développement qu'il conviendrait pour apparaitre comme essentielle. L'idée reprend, tout en les affinant et les contextualisant, les propositions très générales de Gell sur l'effet d'" enchantement " que favorise la virtuosité technique ("The halo-effect of technical 'difficulty'", in Gell, 1994 : 46). Ici, l'émergence de qualités proprement esthétiques et morales laisse place à la perception d'une efficacité particulière. Cette efficacité découle de deux aspects proprement techniques du tatouage: 1) la nature singulière de l'"action outillée " (une percussion perpendiculaire punctiforme avec percuteur) qui semble conférer aux outils une force propre ; 2) la coordination des assistants dans la présentation de la surface à tatouer. La combinaison de ces deux aspects assure la parfaite ré-

7. Voir le livre de Julien Clément (2014) pour l'analyse fine de tels processus à partir, cette fois, de la pratique du rugby.

8. Sans recours à une « iconologie des marques et une sémiotique corporelle " (p. 9), comme c'est le cas, indique S. Galliot, dans la plupart des études sur le tatouage polynésien ou autre. 
alisation de l'action entreprise sans qu'il soit possible, pour les spectateurs, d'en bien comprendre le processus. Cette action est d'autant plus impénétrable qu'elle ne fait l'objet d'aucune forme d'explicitation ou de transmission orale dans une société où, pourtant, l'art oratoire et les élaborations langagières sont essentiels et omniprésents. Cette réflexion se nourrit d'une étude poussée des "modalités praxéologiques, c'est-à-dire du rôle de la construction des savoirs dans la pratique ». Elles forment « les identités professionnelles " des "spécialistes tatoueurs" (p. 10), avec les techniques et "les matérialités " de l'action rituelle. Sont abordées à cette occasion les questions d'adaptation en contexte de changement social, de liberté dans la création artistique, mais aussi les questions de perception des œuvres et de leur efficacité.

Comme on ne voit, la riche ethnographie de Sébastien Galliot ouvre sur d'intéressantes analyses et les propositions théoriques qui en découlent. Elle tend à montrer une réelle perméabilité, du point de vue des Samoans, entre action technique et action rituelle. L'auteur montre l'étroite articulation entre technique et rituel (un complexe "technico-rituel»). Peutêtre pourrait-on aller plus loin et y voir le résultat d'une action nativement "technique-et-rituelle": hors de ses dimensions relationnelles, c'est-à-dire des conceptions spécifiques du monde et de l'action qui en découle, pas de "tatouage " péa, malu, maaliofie, mais des inscriptions sans valeur sur la peau. Sans doute, certains principes techniques sont-ils communs (insérer des pigments sous le derme pour créer une image), mais en aucun cas les pratiques ne se superposent : les tatouages rituels combinent, en une même action, technique et rituel. La plupart des cas étudiés me semblent conforter cette spécificité, sans que cela invalide pour autant le cour des propositions de S. Galliot. L'auteur montre très bien que, au-delà de ce qui se passe dans un registre qu'il considère comme proprement technique, la personne tatouée est le centre d'un réseau à la fois spatial et temporel dense : mobilisation des ancêtres, des déités et de Dieu, des anciens de la communauté et des jeunes appelés pour servir les officiants, des parents et des amis réunissant compétences, argent et biens de valeur nécessaires à l'opération. Bien peu de ce qui se passe là dépend directement du "patient " tatoué, si ce n'est son endurance au mal et le respect des interdits qui visent à le protéger de l'influence néfaste de certains " esprits " aitu. Bien peu, aussi, dépend du tatoueur en définitive, puisque l'abandon ou l'échec de son action (effacement du trait, complications médicales voire décès...) ne résulte pas d'un caprice ou d'une erreur de sa part, mais révèle les relations dysfonctionnelles et la négligence de leurs obligations par les proches du patient. Autrement dit, l'action (l'impression de l'image dans la peau) est ici collective, partagée et diffuse: le tatoueur tatoue, mais la réussite de l'opération dépend un peu du patient et beaucoup de l'état des relations où il trouve sa place. L'auteur souligne à de nombreuses reprises l'importance des configurations relationnelles en jeu dans cette pratique, qưil s’agisse des relations généalogiques avec ses fondateurs, des relations de service mutuel qu'elle crée avec le chef commanditaire, des rapports ambigus avec les esprits qu'il s'agit de contrôler.

Le tatouage apparait donc comme une technologie rituelle centrée sur l'élaboration des relations sociales constituant le monde samoan tel qu'il est conçu, et tel qu'il évolue. Cette étude de cas montre parfaitement que les rites sont essentiellement des techniques réalisant les sociétés en travaillant les relations sociales. Mais ici, l'action technique est rituelle car toute action puise son efficacité dans l'ordre du cosmos, opérant de façon plus ou moins large sur les relations qui composent le monde samoan et sur son organisation. Autrement dit, le tatouage samoan est rituel et en même temps technique parce que dans le monde samoan, toute action efficace ne peut être menée à bien qu'en relation avec les ancêtres (et Dieu), et en accord avec l'ordre du monde afin de le reconduire dans ses grands principes et ses valeurs fondamentales. Il s'agit bien sûr là d'une entreprise exceptionnelle, comme la construction d'une maison, d'une pirogue, autrefois la guerre et les voyages en mer auxquels le chef de la communauté, le matai de la maisonnée, le prêtre, ou le pasteur apportent leur garantie. Bien qu'à un moindre degré, il en va de même pour les actes du quotidien - tels la prise de repas, un match de rugby ou un voyage en car, par exemple. Certes les choses changent, comme change la conception des Samoans sur le monde et sur l'action, plus ou moins autonome, qu'ils peuvent y entreprendre.

On ne peut donc pas manquer de se demander avec Sébastien Galliot si cette spécificité résistera, face aux contraintes sanitaires, à la standardisation des outils, à la dispersion des groupes et aux attentes de générations plus soucieuses d'affirmation identitaire que des responsabilités qu'un tatouage suppose en termes de "service " auprès des anciens et de la chefferie ou de dépendance envers un vaste réseau de vivants et d'ancêtres. Peut-être une étude complémentaire des prestations rassemblées à l'occasion des tatouages documentés par l'auteur (en particulier sur l'origine et la circulation des nattes fines et leur éventuel remplacement par du numéraire ${ }^{9}$ ) permettraitelle de mieux comprendre dans quelle mesure il est désormais possible de se dispenser du soutien de la parentèle pour se faire tatouer lorsqu'on est samoan ? S. Galliot intègre avec raison le tatouage rituel (un " microsystème technique ") dans un "système technique [et rituel] » plus vaste comprenant la préparation des pigments, des outils, des colliers... (p. 390). Une telle analyse pourrait-elle s'étendre, suivant les mêmes principes, aux autres rites du cycle de vie? Oui, semble-t-il. L'auteur éclaire avec justesse les rites du tatouage en faisant appel aux rites de naissance, 
notamment pour ce qui concerne la constitution de l'enveloppe corporelle. Il serait intéressant de voir déployée, dans un travail complémentaire, une étude du cycle de vie où le tatouage s'insère parmi les autres passages. $\mathrm{La}$ " circoncision " et les funérailles des anciens chefs sont mentionnées, mais quelle est la place du tatouage au regard des passages catholiques (pour ceux qui le sont) et les investitures (pour ceux qui y accèdent) qui viennent en complément de la naissance et du mariage par exemple ${ }^{10}$ ? Une perspective longitudinale, saisissant la configuration et les évolutions du réseau relationnel mobilisé lors des différentes cérémonies concernant une même personne, permettrait peut-être aussi d'approfondir l'étude des processus de subjectivation en relation avec les formes rituelles de l'intégration sociale. Bref, positionner le "tatouage rituel " dans le système " technique-et-rituel » plus vaste régissant encore les rapports entre vivants tels qu'ils sont désormais pensés dans leur relation avec le monde, y compris avec les ancêtres et l'au-delà (le monde sociocosmique), pourrait avoir deux intérêts s'il fallait prolonger la passionnante étude menée par $S$. Galliot : 1) celui de mieux comprendre sa permanence au cours des XIX ${ }^{e}$ et $\mathrm{XX}^{\mathrm{e}}$ siècles ; 2) celui de conforter l'analyse proposée selon laquelle l'expertise des maittres tatoueurs et leur connexion généalogique avec les esprits assurant la réussite du rituel sont mises au service de la reconduction sociale dont la chefferie reste responsable. Car c'est, comme l'annonce S. Galliot (p. 30), dans cette connexion que le rituel trouve à la fois son « ancrage physique » et l'essentiel de son efficacité.

En somme, le livre réalise précisément le projet annoncé dans son sous-titre: " un rite polynésien dans l'histoire " en ce qu'il retrace la dynamique propre à une pratique qui perd progressivement une part des dimensions rituelles qui la rendait essentielle dans un cadre sociocosmique pour acquérir d'autres propriétés, tout aussi rituelles semble-t-il, mais dans le cadre, cette fois, de l'engagement personnel en contexte de recompositions culturelles et identitaires propres à une région en mutation. $\mathrm{Ce}$ faisant, il documente avec une rare précision et une grande pertinence l'évolution des pratiques, laissant voir les changements de la société et sa transformation sous l'effet conjugué de la globalisation et de la projection extraterritoriale d'une bonne part de sa population sous forme de communautés recomposées. Il apporte également une analyse précieuse des phénomènes contemporains de subjectivation où le corps et l'attention constante au positionnement statutaire dans une gradation contextuelle - c'est-àdire constamment redéfinie - sont centraux. Enfin, il montre combien une analyse à la fois historique, technologique et praxéologique des rituels est productive, articulant l'étude de l'organisation et des effets de l'action sur la matière et dans l'esprit des observateurs, avec celle des logiques sociales où cette action trouve à la fois un sens et sa pleine efficacité.

\section{RÉFÉRENCES CITÉES}

Chave-Datoen Sophie, 1997. Étude de la natte ta'ovala à Wallis (Polynésie occidentale), Cahiers de linguistique sociale: Y. Broutin (éd.), Se vêtir pour dire, pp. 61-75.

—, 2013. L'incorporation des déités polynésiennes. Mimèsis, actualisation, instanciation : les modalités de la représentation. L'Homme. Revue française d'anthropologie 205, pp. 55-78.

CLÉment Julien, 2014. Cultures physiques. Le rugby de Samoa, Paris, éditions Rue d'Ulm.

GeLl Alfred, 1993. Wrapping in images. Tattooing in Polynesia, Oxford, Clarendon Press.

- 1994 . The technology of enchantment and the enchantment of technology, in Jeremy Coote (ed.), Anthropology, Art, and Aesthetics, Oxford, Clarendon Press, pp. 40-63.

—, 1995. Closure and Multiplication. An Essay on Polynesian Cosmology and Ritual, in Daniel de Coppet \& André Iteanu (eds), Cosmos and Society in Oceania, Oxford-Washington, Berg, pp. 21-56.

Mauss Marcel, 1950. Essai sur le don, in Sociologie et anthropologie, Paris, PUF, pp. 145-279.

Tcherkezoff Serge, 2003. Fa'a-Samoa. Une identité polynésienne (économie, politique, sexualité). L'anthropologie comme dialogue culturel, Paris, L'Harmattan.

—, 2013. La valeur immatérielle des nattes fines de Samoa : une monnaie au sens maussien, Journal de la Société des Océanistes 136-137, pp. 43-62 (https://doi.org/10.4000/jso.6905).

Sophie Chave-Dartoen, Université de Bordeaux

10. Un lien est mentionné entre le tatouage et une seconde naissance (notamment la cérémonie de clôture samaga et un autre baptême, p. 365 ; voir p. 366 pour un parallèle rapide avec les investitures). La question des conceptions locales de la personne, de sa constitution et de son traitement, quotidien et plus particulièrement rituel, pourrait être utilement approfondie. 